\title{
Effect of Modeling Techniques on the Simulation: Calculating the Stress Concentration Factors in Square Hollow Section T-Joints as a Case Study
}

\author{
Serwan S. M.Talabani ${ }^{1}$, Ahmad T. Azeez ${ }^{1}$, Silvio D. Barros ${ }^{2,3}$, Basim M. Fadhil ${ }^{1}$ and Hewa H. Omer ${ }^{4}$ \\ ${ }^{1}$ Department of Manufacturing and Industrial Engineering, Faculty of Engineering, Koya University, \\ Koya KOY45, Kurdistan Region - F.R. Iraq \\ ${ }^{2}$ Federal Center of Technological Education in Rio de Janeiro - CEFET/RJ, \\ Rio de Janeiro, Brazil \\ ${ }^{3} \mathrm{GeM}$ Institute, UMR 6183 CNRS, CESI, \\ Saint-Nazaire, France \\ ${ }^{4}$ Department of Chemical Engineering, Faculty of Engineering, Soran University, \\ Kurdistan Region - F.R. Iraq
}

\begin{abstract}
The capability of the simulation software motivates the engineers to analyze the stress in complex welded joints where ordinary mathematical expression may lack. However, unfortunately, the simulations were interpreted mechanically without considering the simulation parameters deeply. For this reason, this research aims to study the effect of simulation parameters on the stress concentration factor of square hollow sections structures using ANSYS workbench. In this context, two main meshing techniques (face split vs. slicing) and various behavior of materials (linear vs. nonlinear) having been considered. The outputs of the investigations revealed the superiority of the materials nonlinearity over linear behavior in terms of results acceptability comparing to their corresponding real-life ones. For instance, the supercritical fluid of nonlinear plastic material estimated to be 14 which was closest to the 12 obtained experimentally by Mashiri et al. In addition to that, face split technique outperformed the slicing in terms of result accuracy and solving time. Solving the face split model elapsed $254 \mathrm{~s}$ only which is dramatically less than the minimum time (1605 s) of sliced models. This is because any slicing process will lead to the formation of the contact elements at interfaces and uncomfortable meshing which may in turn adversely effect on the simulation efficiency.
\end{abstract}

Index Terms-ANSYS Workbench, Face split, Modeling of materials, Stress concentration factor, Welding toe.

\section{INTRODUCTION}

In modern life, due to the great development in the field of the computers, the engineers rely to a large extent on simulating

ARO-The Scientific Journal of Koya University

Volume VIII, No.1 (2020), Article ID: ARO.10585, 7 pages

DOI: $10.14500 /$ aro. 10585

Received: 03 November 2019; Accepted: 23 June 2020

Regular research paper: Published: 28 June 2020

Corresponding author's email: serwan.talabani@koyauniversity.org

Copyright (C) 2020 Serwan S. M. Talabani, Ahmad T. Azeez,

Silvio de Barros, Basim M. Fadhil, Hewa H. Omer. This is an open-access article distributed under the Creative Commons Attribution License. virtual physics in a circumstance comparative to their real-life conditions. Accordingly, simulation is a crucial instrument to obtain immediate results of the models operating under different working conditions. This contributes to shortening the design session and also enables the engineers to review, manipulate, and improve their designs continuously at low levels of cost (Sinha, et al., 2000) For this reason, different simulation programs were innovated to cover the specific scientific requirements of different engineering disciplines. For example, ANSYS which is a multiphasic package developed by ANSYS Inc., USA, and has been widely used by engineers due to the enjoyed capability and has different products such as Axiomatic Product Development Lifecycle and WORKBENCH.

On the other hand, welding is an effective method for assembling parts together, especially in construction applications such as bridges, towers, and offshore platforms. Such structures, considering their purpose of the building may be vulnerable to in situ loading/unloading cycles originated from traffic loads, winds, or sea waves, leading to fatigue damage (Saini, Karmakar and Chaudhuri, 2016). The dynamic load may initiate crack at welded joints which may propagate resulting in the collapse of the structures eventually. The abnormal increment of the stresses at welded beads related to the discontinuity at the joints is the main source of crack initiation (Zhao, et al., 2001). Thus, considering the supercritical fluids (SCFs) in the detailed design is a top concern. According to the recommendations for fatigue design in welded joints, the SCFs, which are the ratio of the hot spot stress $\left(\sigma_{h s}\right)$ to the nominal stress, can be calculated either practically or numerically as these are the only applicable methods to determine the $\sigma_{h s}$. Unlike, the nominal stress is covered by simple beam theory (Hobbacher, 2008). 
Calculating SCFs numerically require qualitative meshing such as fine meshes around the weld toe to improve data capture and coarser elements elsewhere to reduce the cost of computations (Zhao, et al., 2001). Consequently, the researchers analyzed the welding joints under different considerations of meshing such as size, type (solid or shell), and shape (hexagonal or tetrahedral) and different conclusions have been made (Zhao, et al., 2001), (Hobbacher, 2008), and (Neimi, Fricke and Maddos, 2004). For instance, it is estimated that solid elements prevailed over shell elements in terms of accuracy unless the shell elements manipulated (Rizzo and Codda, 2010). Subsequently, a set of rules to mesh the model with large shell elements have been developed by Chattopadhyay (Chattopadhyay, et al., 2011) and the new formula enabled the calculation of the SCFs effectively. In the same context, numerous researches refined the elements around the welding toe without any refereeing to the effect of the meshing technique on the performance of the simulation in terms of time or the results accuracy. In many cases, the literature did not explain the technique used in mapping the models and the researchers concentrated on discussion their works from a pure mechanical point of view such as Tong (Tong, et al., 2016), Chiew (Chiew, et al., 2000), and (Chiew, et al., 1999) without any attention to the modeling part.

It is obvious from the literature that the effect of the meshing techniques on the simulation performance is by far still obscure and needs to be demonstrated. In accordance, this paper aims to study two meshing techniques (slicing and face split) provided by ANSYS workbench in mapping the elements in square hollow section (SHS) welded tubes and investigate their effects on the simulation performance. In addition, the effect of the material was also included to cover this subject from its different aspects. The paper will also verify the result of the SCFs by considering the real life behavior of the welded structures cited in the literatures.

\section{Methodology}

\section{A. Calculating Stress Concentration Factors (SCFs)}

SCF is a multiplication factor of the stress around discontinuous regions, defects, crack, etc., and welding joints are not excluded. Dividing the $\sigma_{h s}$ by the nominal stress will determine SCFs at welding toes which depend on different dimensionless parameters. Unfortunately, the available analytical methods are incapable of calculating the $\sigma_{h s}$ on the surface of the tubes, hence, the engineers depend on the practical and/or numerical method to determine the structural stress (Saini, Karmakar and Chaudhuri, 2016), (Zhao, et al., 2001), and (Hobbacher, 2008). The numerical method depends on extrapolation the stresses at particular points from the weld edge linearly for circular tubes or quadratically for SHS [2]. The distances of the extrapolation points in the SHS with their minimum limits are given in Table I, as recommended by Zhao et al. (Zhao, et al., 2001). Experimentally, it is feasible to estimate the strains pointed at specific distances using a special type of chips known as strain gauge. For this reason, strain concentration factor will be determined first then it will be converted to SCF using the following formulas:

$\mathrm{SCF}=1.1 \mathrm{SCNF}$ for rectangular hollow sections (RHSs)

$\mathrm{SCF}=1.2 \mathrm{SCNF}$ for circular hollow sections (CHSs)

Furthermore, it is important to notice that rising of the stress in the extrapolation region has a negatively concaved scheme, as shown in Fig. 1.

\section{B. Finite Element Method}

Finite element is an active method for solving differential equations and analyzing complex systems in engineering and physics where the ordinary analytical methods are incapable of solving the problems. The method consists of discretizing the virtual prototype into definite parts with a specific number of nodes based on the shape of the element. Each individual part has a stiffness matrix which will be assembled to the global stiffness matrix following a particular algorithm. An example of such matrix may be found in Equation 1.

$$
\{\mathrm{F}\}=[\mathrm{K}]\{\mathrm{d}\}
$$

$\{\mathrm{F}\}$ : Global nodal force matrix

$[\mathrm{K}]$ : Global stiffness matrix

$\{d\}$ : Global nodal unknown displacement matrix

The size of the previous matrices depends on the total number of unknown degrees of freedom (DOF) of the nodes in the model which, in turn, depends on the number of nodes. Investigating the PCS files provided by ANSYS revealed the fact that the total number of DOF is 3 times the number of nodes recalling that three-dimensional (3D) solid being used in meshing. Off course, this is not a general trend to be globalized fundamentally, actually, the aforementioned trend of DOFs depends on the types of the elements used in

TABLE I

Boundary Condition of the Extrapolation Region [3]

\begin{tabular}{lccc}
\hline \hline Distance from welding toe & Chord & Brace & Minimum limits \\
\hline Lr,min & $0.4 \mathrm{t}_{0}^{\mathrm{a}}$ & $0.4 \mathrm{t}_{1}^{\mathrm{b}}$ & $4 \mathrm{~mm}$ \\
Lr,max & Lr,min $+\mathrm{t}_{0}$ & Lr,min $+\mathrm{t}_{1}$ & Lr,min $+0.6 \mathrm{t}_{1}$ \\
\hline \hline
\end{tabular}

$\mathrm{a}$ and $\mathrm{b}$ are the thickness of the chord and brace, respectively

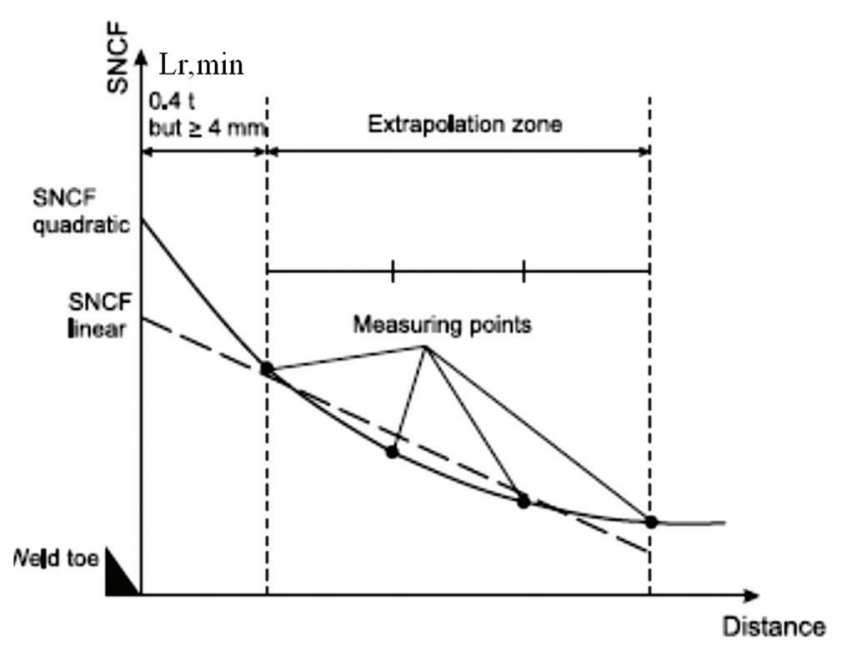

Fig.1. Extrapolation methods (Zhao, et al., 2001). 
meshing and might be restricted to the models used in this research, see the upcoming sections for details. Accordingly, simulating 3D models reflect adversely on the elapsed time (which is the time required by the software to solve the matrices and obtain the results) due to DOFs factor explained previously. In the same context, it is sensible to say that any meshing refinement leads to increment in the running time of the simulation since the matrices size enlarge in combination with the number of DOF (Bhashyam, 2002).

On the other hand, slicing off the models to control the partitioning process will lead to the formation of the contact elements at the interface. Such contact pairs need a specific algorithm that controls the interaction of the physics at interfaces in a manner similar to their real-life behavior. Consequently, various formulations are available such as pure Penalty and Augmented Lagrange which have the following form (Contact Formulation Theory, 2016) and (Stupkiewicz, 2003).

$$
\text { Pure Penalty } \mathrm{f}_{\mathrm{n}}=\mathrm{k}_{\mathrm{n}} \times \text { penetration }
$$

Augmented Lagrange $\mathrm{f}_{\mathrm{n}}=\mathrm{kn} \times$ penetration $+\lambda$

$\mathrm{f}_{\mathrm{n}}$ : Finite contact force

$\mathrm{k}_{\mathrm{n}}$ : Contact stiffness matrix

$\lambda$ : Lagrange multiplier vector defined on the contact surface. It is thought that the presence of contact pairs in the models consumes time since the software has to deal with forces transfer between the nodes at interfaces according to the contact formulas.

\section{Modeling AND Simulation}

\section{A. Modeling of Materials}

Materials modeling means selecting the most consistent mathematical expressions in the simulation that keeps the response of the materials to the various working conditions in a context to the physical evidence (Haupt, 1993) otherwise the simulation will be useless. Accordingly, different behaviors were used to investigate the effect of the materials modeling on the SCFs and the elapsed time. The three known models, linear, bilinear, and multilinear, were tested. The modulus of elasticity and Poisson's ratio for steel were taken as $205 \mathrm{GPa}$ and 0.3 , respectively. The curves of different materials behavior are shown in Fig. 2. (Liu and Day, 2009). The tangent modulus was calculated manually from the multilinear data and estimated to be $868 \mathrm{MPa}$.

\section{B. Meshing Control and Boundary Condition}

The intact meshing of the objects to the specific map may be obstructed by the complexity of the models unless advanced techniques utilized. Two of the advanced methods to control the meshing in ANSYS workbench are face split and slicing. Face split, as the name suggests, depends on splitting the faces without cutting off the object through the thickness; hence, no contact will be formed at the splitting interfaces. Slicing, on the other hand, relies on partitioning the model into smaller parts.

Despite that, slicing provides the users the facility of controlling meshing process, especially in complex geometry, but the process is not free of risks. Models being sliced have a new physical state at interfaces due to the formation of contact elements which in turn may result in convergence problem especially when nonlinear materials having been adapted in the simulations (Barrett, 2014). However, the elements around the weld toe were sized to $1 \times 1 \mathrm{~mm}$ and approximately $20 \mathrm{~mm}$ width faraway, the welding profile has been concaved with $6 \mathrm{~mm}$ diameter to oversize the weld bead as recommended by Mashiri [18], Fig. 3a.

Regarding boundary condition, a $2 \mathrm{kN}$ in-plane lateral force was applied on the top surface of the brace of dimensions $50 \times$ $50 \times 3 \mathrm{~mm}$, whereas the $100 \times 100 \times 3 \mathrm{~mm}$ chord constrained from its two side faces, Fig. 3b. The length of each of the chord and the brace was 6 times their widths (Tong, et al., 2016). The previous specific dimensions of the tubes were selected to verify the resulted SCFs with its corresponding practical value obtained by Mashiri (Mashiri, et al., 2007). Unfortunately, slicing produced uncomfortable meshing as can be seen in Fig. $3 \mathrm{c}$ and $d$. Such meshing may have detrimental effects on the simulation efficiency. The nodes in uncomfortable meshing may be brought into intimated contact using node merging option available in ANSYS workbench 16.1 release. Each node merging process contributes to reducing the total degree of freedom of the model which,
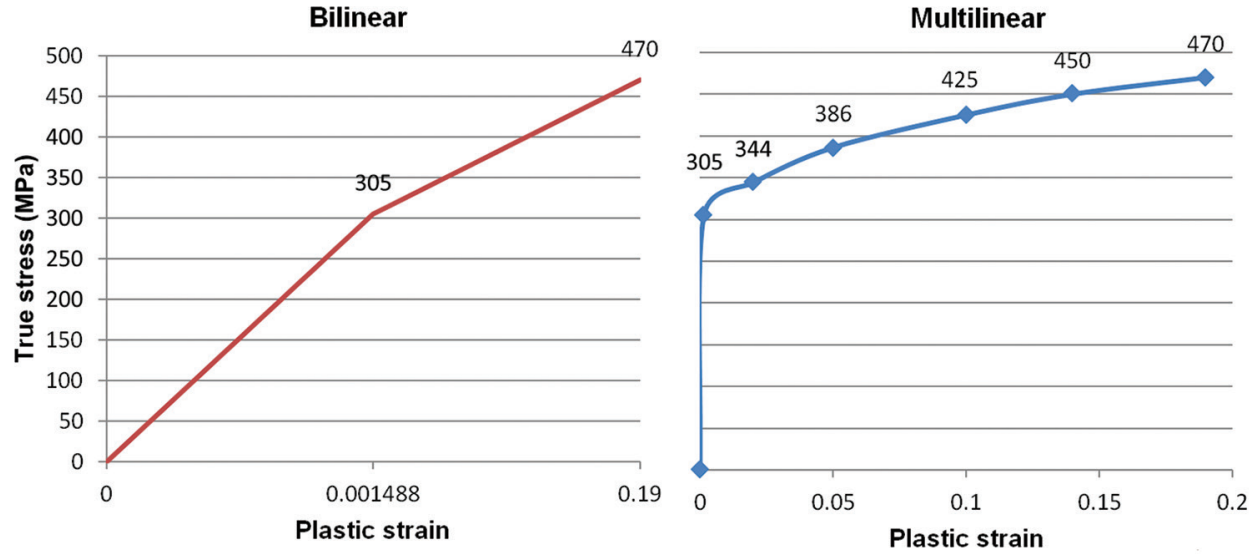

Fig. 2. Models of materials (the experimental data cited from Liu and Day, 2009). 
in turn, affects the simulation process. Accordingly, four models were tested in order to evaluate the performance of the simulation considering meshing robustness, each with a specific degree of freedom resulted from merging the nodes (model 1-model 4, table II). The technique of meshing itself (face split vs. slicing) will be criterion to complete the evaluation of the simulation efficiency. Not to mention that hexagonal elements with dimension $1 \times 1 \mathrm{~mm}$ around the weld toe and $1 \times 20 \mathrm{~mm}$ elsewhere being used to mesh the models as explained previously.

\section{Results AND Discussion}

\section{A. Materials Modeling}

The hot spot stress estimated by extrapolating the maximum principal stresses quadratically within the limits 4 and $7 \mathrm{~mm}$ from the weld toe according to the welding design criteria recommended to select the boundary of the extrapolation region required to calculate the $\sigma_{h s}$, as indicated in Table I. The method of extrapolation was developed in the 1970s to calculate the $\sigma_{h s}$ raised on the surface of the welded structures due to uneven levels of deformations in brace and chord and to distinguish it from notch stress which is a local stress originated from the notch of the weld toe (Saini, Karmakar and Chaudhuri, 2016) and (Zhao, et al., 2001). The value of the extrapolation region should also be $3 \mathrm{~mm}$ away from the corner where the maximum stress occurred to avoid the singularity as advised by Wingerde (Wingerde, 1992), Fig. 4a. The third point could be selected arbitrarily between them (Mashiri, et al., 2002) and it was selected at a distance of approximately $6 \mathrm{~mm}$ away from the weld edge.

SCFs were calculated in different models through quadratic extrapolation using Microsoft巴 Excel sheet. The value of $\sigma_{h s}$ could be simply estimated by substituting $\times$ with zero in the trend line equation indicated in Fig. 4b. The $\sigma_{h s}$ then divided by the nominal stress (16.4 MPa) calculated manually in the chord using simple beam theory.

Moreover, the effect of different materials behavior on the SCFs and elapsed time is shown in Fig. 5. Modeling the material multilinearly best fitted the real-life behavior as expected because the calculated SCF 14 was closest to the practical value 12 estimated by Mashiri (Mashiri, et al., 2007). Unfortunately, it is not possible to obtain the same SCF as in the referred work because the authors did not provide any stress-strain data that could be used in here. The SCFs in both linear and bilinear modeling diverged from the practical value and exceeded 17, as shown in Fig. 5.

Regarding the elapsed time, a variant trend was observed as the running time increased dramatically from $254 \mathrm{~s}$ to more than $3500 \mathrm{~s}$, as shown in Fig. 5, due to the increment of the total iterations in preconditioned conjugate gradient from 1453 to more than 2000 in linear and nonlinear (bilinear and multilinear) models, respectively. However, this is not always the case, because if multiple cores of CPU are used (parallel processing), the nonlinearity will increase the time required to solve one iteration rather than the number of iterations itself (Koslpski, 2017). The elapsed time may vary from one machine to another based on the capability of the system, but the obtained trend plotted in Fig. 5. is expected to remain the same.
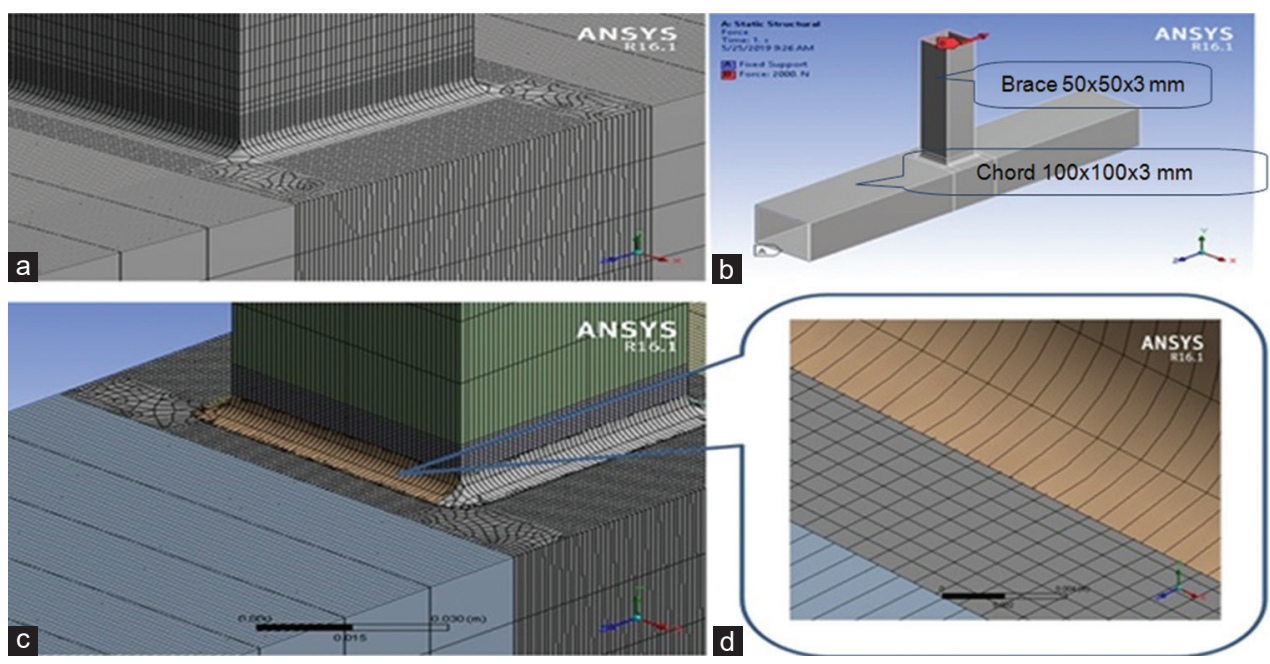

Fig. 3. Meshing techniques and boundary conditions. a. Face split b. Boundary condition c. Sliced technique d. Uncomfortable meshing.

TABLE II

Effect of Meshing Techniques on the Running Time

\begin{tabular}{|c|c|c|c|c|c|}
\hline \multirow[t]{2}{*}{ Criteria } & \multirow[t]{2}{*}{ Face split } & \multicolumn{4}{|c|}{ Sliced models } \\
\hline & & Model 1 & Model 2 & Model 3 & Model 4 \\
\hline Elapsed time (s) & 254 & 2792 & 2773 & 2750 & 1605 \\
\hline Number of nodes & 25,0671 & 199,064 & 198,865 & 198,764 & 198,727 \\
\hline Degree of freedoms & 752,013 & 597,192 & 596,595 & 596,292 & 596,181 \\
\hline Non-zeros in upper triangular part of global stiffness matrix & 752,013 & $40,559,457$ & $40,514,532$ & $40,488,627$ & $40,476,282$ \\
\hline
\end{tabular}




\section{B. Effect of Meshing Techniques}

The recommended meshing map for calculating SCFs in welded joints can be controlled using advanced technique such as face split or slicing. Slicing, as explained previously, will result in interface at the cutoff planes and sometimes uncomfortable meshing at contact boundary of the parts that may have detrimental effects on the simulation performance. Consequently, four models, each one with a specific number of nodes produced due to slicing, were investigated and compared to one-part object in which the meshing process was controlled through face splitting.

To reduce the running time and avoid the convergence problems related to the contact nonlinearities of the sliced models, the material behavior was modeled linearly and compared to their corresponding linearly deformed material in face split models. The SCFs, for instance, even could not be evaluated in sliced models since the stress in the extrapolation region concaved positively in contrast to the standard trend, as can be seen comparing Fig. 6. with Fig. 1. This leads to $\sigma_{h s}$ of magnitude $399 \mathrm{MPa}$ at weld toe which is lower than $475 \mathrm{MPa}$ determined at a distance $4 \mathrm{~mm}$ from welding edge and of course such result is unacceptable.

Fortunately, the stress in the linear model meshed using face split concaved up in agreement to the standard style and the SCF was calculated to be 17 , as discussed in the previous section. The reason may be due to the differences in the meshing style through the thickness (as shown in Fig. 7) despite their similarity on the surface as ANSYS meshed the sliced model piece wisely whereas the face split as one part.

On the other hand, uncomfortable meshing produced in the sliced models adversely affected the elapsed time as the number of nodes increased. Subsequently, merging nodes (a technique that brings the nodes into intimate contact at interfaces) plays a key role in reducing the number of nodes hence improves the performance of simulation in terms of running time (Bhashyam, 2002).
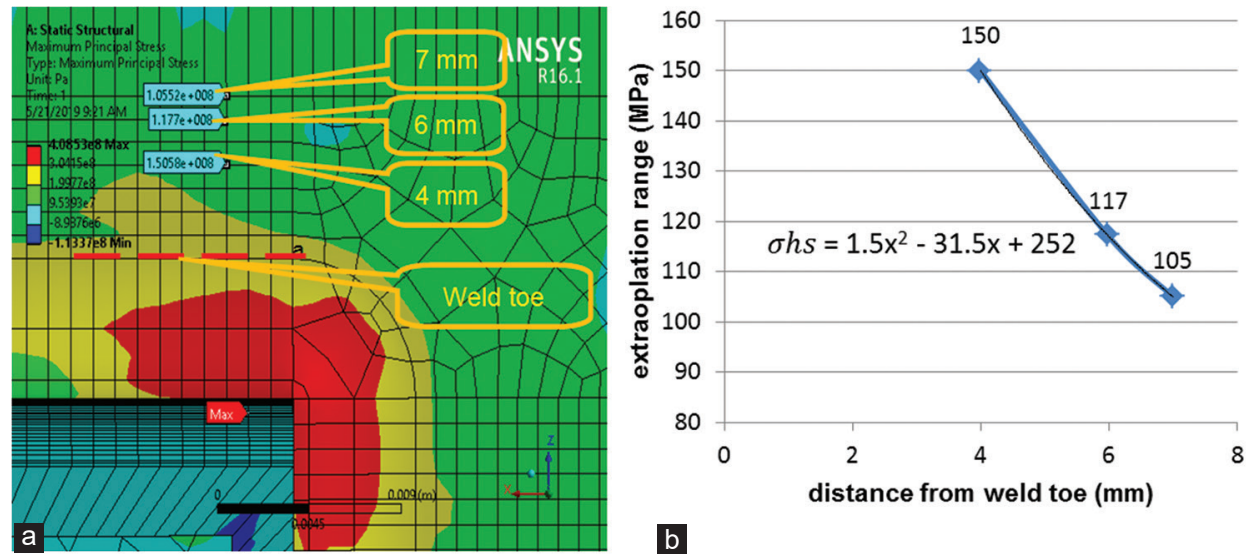

Fig. 4. Calculating the hot spot stress a. Maximum principal stress on the chord surface at the location of maximum square b. The result of the extrapolation.

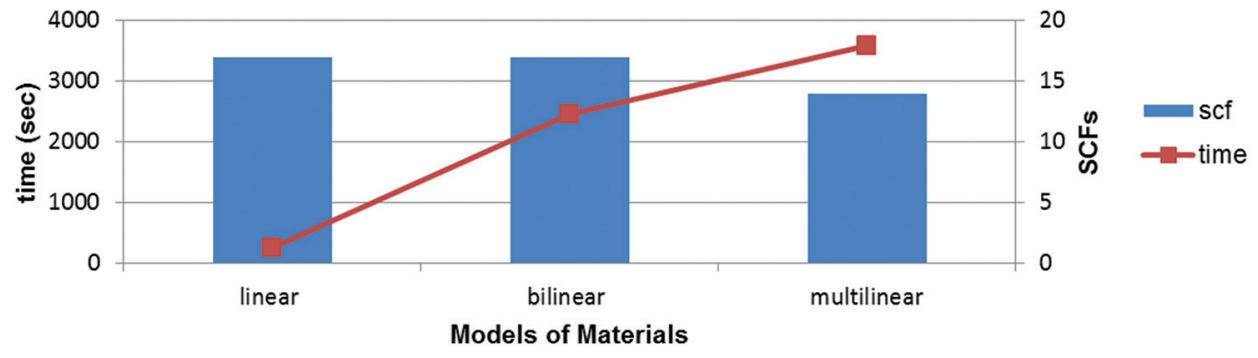

Fig. 5. Effect of materials behavior on the supercritical fluid and elapsed time.

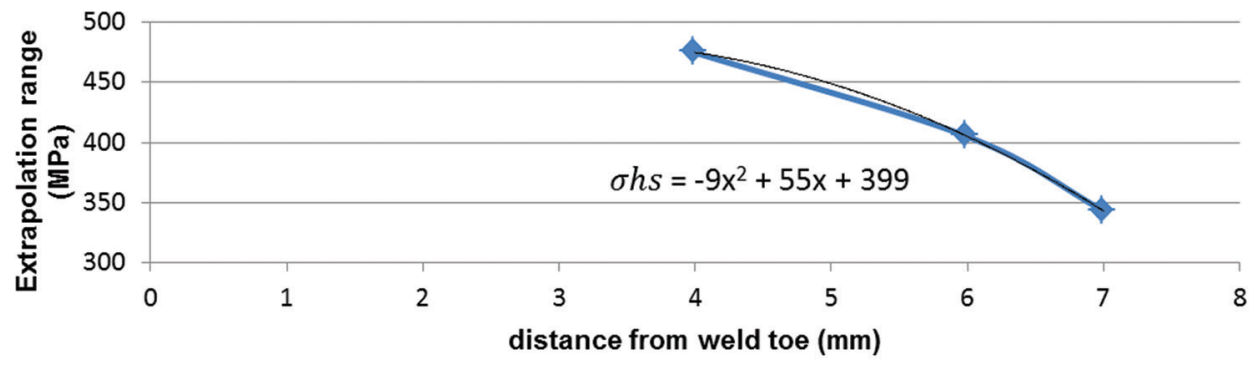

Fig. 6. Convex extrapolation region in the sliced model of linear material. 


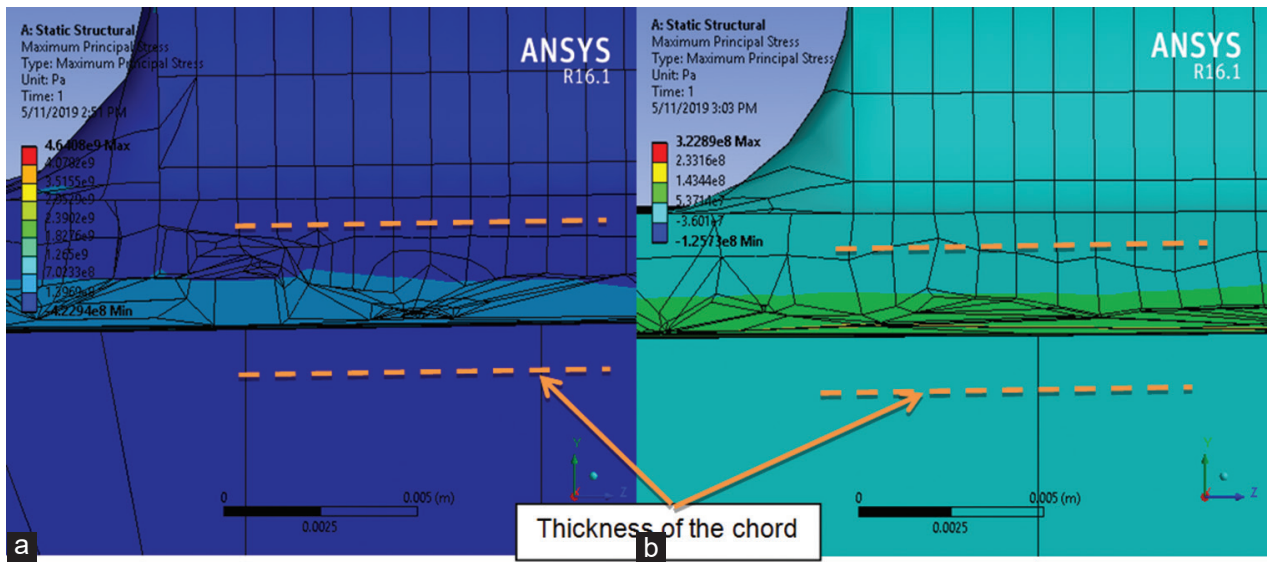

Fig. 7. The differences in the meshing through the thickness. a. Sliced model, b. Face split model.

The elapsed time reduced by a parallel reduction in the number of nodes from $2792 \mathrm{~s}$ to $1605 \mathrm{~s}$ as the number of nodes decreased from 199,064 to 198,727 in models 1 and 4 , respectively, by merging nodes to produce comfortable meshing. The obtained results agreed to the mathematical expressions provided by Equation 1. The lower the number of nodes, the lower the degree of freedom related to the models hence lower non-zeros numbers that ANSYS has to solve, Table II. Unfortunately, additional reduction in the number of the nodes was not possible since any further nodes merging would produce highly deformed elements that did not converge during solving probably due to poor meshing quality.

However, comparison with face split may change the aforementioned discussion. Despite of that face split model contains 250,671 nodes, the elapsed time was only 254 $\mathrm{s}$ compared to more than $1600 \mathrm{~s}$ for sliced models due to the presence of the contact elements in the later. In sliced models, ANSYS has to solve additional equations related to contact stiffness matrix. For example, the face split contains 752,013 non-zeros in the global stiffness matrix compared to more than 40 million factors in other models. The additional non-zeros factors may be originated from the contact stiffness matrix and decrement of such non-zeros coefficients through reduction of the degree of freedoms in the sliced models by merging nodes also support such an explanation, Table II.

It is clear from the previous discussion that face split outperformed slicing technique due to inaccurate-high cost for the later. However, slicing is still an efficient tool of meshing if the disadvantages of the process avoided. For instance, considering the contact problems and the robustness of the meshing at the interface are the main criteria to be considered.

\section{CONCLUSION}

The paper investigated the effect of different parameters such as materials behavior and meshing techniques on the results of simulations using ANSYS software. The previous discussions led to important conclusions regarding the simulation process and software behavior that can be summarized as follows:
1) Simulation the material nonlinearly though its timeconsuming process but best verified the resulted SCF as compared to the real-life value.

2) Face split unless the problematic factors have been avoided outperformed the slicing technique in terms of results accuracy and running time.

3) Presences of contact elements in the sliced models adversely affected the simulation efficiency and dramatically increase elapsed time as the number of degree of freedom increased.

\section{FUTURE WORK}

The paper investigated the effect of various parameters on the simulation results in welded joints using ANSYS WORKBENCH. However, the output of the work disclosed completely new gaps to be covered in extended works. For example, comparing the obtained results from another software rather than ANSYS with that cited in this paper. However, the authors recommend comparing under the same working conditions as element size, type, and number, the model design and dimensions, and the material data to guarantee a pure attribution of the results on the software type. On the other hand, estimating the residual stress in welded joints is another factored to be considered in the future.

\section{REFERENCES}

Barrett, P. 2014. Stress Analysis Convergence Tips for Dummies. Available from: https://www.caeai.com/blog/stress-analysis-convergence-tips-dummies. [Last accessed on 2019 May 24].

Bhashyam, G.R. (2002) ANSYS Mechanical A Powerful Nonlinear Simulation Tool. ANSYS, Inc., Canonsburg, Pennsylvania.

Chattopadhyay, A., Glinka, G., El-Zein, M. and Qian, J. 2011. Stress analysis and fatigue of welded structures. Welding in the World, 55(0708), pp.1-21.

Chiew, S.P., Soh, C.K., Members, A.S.C. and Wu, N.W. 1999. Experimental and numerical stress analysis XT-joint. Journal of Structural Engineering, 125, pp.1239-1248.

Chiew, S.P., Soh, C.K., Members, A.S.C. and Wu, N.W. 2000. Experimental and numerical SCF studies of multiplanar tubular XX-joint. Journal of Structural Engineering, 126, pp.1331-1338. 
Contact Formulation Theory. 2019. Available from: https://www.sharcnet.ca/

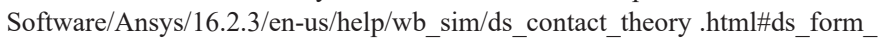
pure_aug. [Last accessed on 2019 May 26].

Haupt, P. 1993. On the mathematical modeling of material behavior in continuum mechanics. Acta Mechanica, 100, pp.129-154.

Hobbacher, A. 2008. Recommendations for Fatigue Design of Welded Joints and Components. International Institute of Welding, Paris.

Koslpski, J. 2017. Speeding up Your Analysis Part 1. Available from: https:// www.caeai.com/blog/speeding-your-analysis-\%E2\%80\%93-part-1. [Last accessed on 2019 May 21].

Liu, Y.C. and Day, M.L. 2009. Concept modeling of tapered thin-walled tubes. Journal of Zheijang University Science A, 10(1), pp.44-53.

Mashiri, F.R., Zhao, X.L., Grundy, P. and Tong, L. 2002. Fatigue design of welded very thin-walled SHS to-plate joints under in-plane bending. Thin Walled Structures, 40, pp.125-151.

Mashiri, F.R., Zhao, X.L., Hirt, M.A. and Nussbaumer, A. 2007. Size effect of welded thin walled tubular joints. International Journal of Structural Stability and Dynamics, 7(1), pp.101-127.

Neimi, E., Fricke, W. and Maddos, S. 2004. Structural Stress Approach to Fatigue Analysis of Welded Components Designer's Guide. International Institute of Welding, Springer, Singapore.
Rizzo, C.M. and Codda, M. 2010. Application of the structural stress approach to a welded joint with complex geometry. Welding International, 24(5), pp.390-400.

Saini, D.S., Karmakar, D. and Chaudhuri, S. 2016. A review of stress concentration factors in tubular and non-tubular joints for design of offshore installations. Journal of Ocean Engineering and Science, 1, pp.186-202.

Sinha, R., Liang, V.C., Paredis, C. and Khosla, P. 2000. Modeling and simulation methods for design of engineering systems. The ASME Journal of Computing and Information Science in Engineering, 1(1), pp.84-91.

Stupkiewicz, S. 2003. Augmented Lagrangian Formulation and Sensitivity Analysis of Contact Problems. VII International Conference on Computational Plasticity, Barcelona.

Tong, L., Xu, G., Yan, D. and Zhao, X.L. 2016. Fatigue tests and design of diamond bird- beak SHS T- joints under axial loading in brace. Journal of Construction Steel Research, 118, 49-59.

Wingerde, A.M. 1992. The fatigue behavior of T and X-joint made of square hollow sections. Heron, 37(2), pp.3-182.

Zhao, X.L., Herion, S., Packer, J.A., Puhtli, R.S., Sedlacek, G., Wardenier, J., Weynand, K., Wingerde, A.M. and Yeomans, N.F. 2001. Design Guide For Circular and Rectangular Hollow Section Welded Joints Under Fatigue Loading. TÜV-Verlag GmbH, Germany. 